\title{
ASPECTOS EPIDEMIOLÓGICOS DA TENIASE-CISTICERCOSE NA ÁREA ENDÊMICA DE LAGAMAR, MG
}

\author{
Mario León Silva-Vergara, Aluízio Prata, Cláudio de Oliveira Vieira, \\ João Henrique Castro, Luciane Giroto Micheletti, Arturo \\ Santana Otaño e João Franquini Júnior
}

\begin{abstract}
Um inquérito epidemiológico sobre teniase-cisticercose foi realizado em LagamarMG em 1992. Cadastradas 1109 casas com 3344 babitantes. O inquérito abrangeu 875 (86\%) familias e foi respondido por um informante que, em $80 \%$ dos casos, foi o pai. Na cidade bavia 100 chiqueiros em $100(11,4 \%)$ casas que albergavam 406 suinos, em condiçôes extremamente precârias. Em 300 (34,2\%) casas bavia o antecedente de teniase em algum membro da familia. O antecedente de convulsẫo foi relatado por 125 (14,2\%) familias. O inicio das convulsões na idade adulta foi caracterizado em 39 $(37,8 \%)$ familias e o antecedente de doenças mentais foi relatado em $53(6 \%)$ casas. Os exames parasitológicos de fezes mostraram ovos de Taenia spp em 24 (1,3\%) das 1850 amostras examinadas.
\end{abstract}

Palavras-chaves: Teníase. Cisticercose. Convulsão. Inquérito epidemiológico.

Segundo estimativas conservadoras da Organização Mundial da Saúde, haveria no mundo, pelo menos, 2,5 milhões de pessoas acometidas por teniase, distribuidas principalmente, na América Latina, antiga URSS, África, India e Extremo Oriente. A teníase-cisticercose está relacionada ao consumo de carne crua ou mal cozida, além de água poluída, hortaliças mal cozidas e frutas contaminadas, sendo perpetuada pela pobreza, falta de educação e precária infraestrutura sanitária, permitindo estreito contato do homem com os suínos. Nessas circunstâncias, a criação dos suínos é realizada, na maioria das vezes, em forma primitiva e a comercialização e consumo da carne são feitos clandestinamente ${ }^{611314}$.

A relevância da $T$. solium é devido ao fato de as larvas Cysticercus cellulosae alojarem-se acidentalmente nos tecidos humanos, especialmente no cérebro, olhos e músculos, causando a cisticercose, produzindo alta morbidade e, às vezes, mortalidade nas formas mais severas ${ }^{1237}$.

A cisticercose cerebral representa uma carga muito onerosa, devido às perdas

Disciplina de Doenças Infecciosas e Parasitárias da Faculdade de Medicina do Triângulo Mineiro, Uberaba, MG. Endereço para correspondencia: Dr. Mário León SilvaVergara. Medicina Tropical/FMTM. Caixa118, 38001-970 Uberaba, MG.

Recebido para publicação em 23/02/95 econômicas decorrentes de hospitalizações, tratamento médico e/ou cirúrgico, ausências laborais pela incapacidade física e mental e transtornos sócio-econômicos do núcleo familiar. A carne dos animais parasitados não pode ser comercializada e consumida pelo menos nos locais onde há controle sanitário ${ }^{11} 13$. Os prejuízos têm sido em geral subestimados, pela ausência de dados estatísticos. Ademais, existem poucos estudos epidemiológicos nas áreas urbanas e rurais remotas, especialmente, quanto aos fatores relacionados à manutenção desta parasitose 8912162324 . Por essa razão, consideramos de interesse realizar um estudo em área de transmissão da doença, para avaliar os fatores epidemiológicos envolvidos na sua transmissão.

\section{MATERIAL E MÉTODOS}

Realizamos inquérito epidemiológico sobre teníase-cisticercose na cidade de Lagamar (MG), região do Alto Paranaíba, distante $300 \mathrm{~km}$ de Belo Horizonte. A economia dessa cidade baseia-se primordialmente na agropecuária. A criação de suínos é prática bastante difundida, tanto na área urbana quanto nas zonas rurais e favorecida pela existência de um laticínio que produz grande quantidade de soro, utilizado como alimentação complementar na criação desses animais. 
Silva-Vergara ML, Prata A, Vleira CO, Castro JH, Micheletti LG, Otaño AS, Franquini Júnior J. Aspectos epidemiológicos da teniase-cisticercose na área endêmica de Lagamar, MG. Revista da Sociedade Brasileira de Medicina Tropical 28:345-349, out-dez, 1995.

O cadastramento da população foi realizado através de visita domiciliar, quando foi preenchida ficha-padrão, com dados fornecidos pelo chefe da família e, na sua ausência, pela pessoa mais qualificada para responder às questões no momento da visita

Nessa ficha, constaram os seguintes dados: identificação do informante, condiçôes da moradia, fonte de água, depósito de lixo, presença de animais domésticos, em especial, suínos, condições e antecedentes de sua criação, antecedentes de teníase, eliminação de proglotes ou vermes adultos, tratamento e antecedentes de cisticercose, como convulsões, doença neurológica ou mental nos membros da família nuclear.

Para o exame parasitológico de fezes da população, foram distribuídas vasilhas em todas as casas e dada orientação quanto à coleta das amostras. Para cada amostra, foram realizados exames pelos métodos de sedimentação (Hoffmann Pons e Janer, KatoKatz e Baermann-Moraes). Procuramos obter informações das autoridades de saúde locais sobre a infra-estrutura sanitária da cidade.

\section{RESULTADOS}

No cadastramento que fizemos em Lagamar, haviam 1109 casas, das quais 91 $(8,2 \%)$ não eram habitações (bares, igrejas, escolas, edifícios públicos, casas comerciais etc), $95(8,5 \%)$ estavam desabitadas e em 48 $(4,3 \%)$ não foram encontrados moradores, em várias visitas realizadas. Assim, restaram 875 $(78,9 \%)$ casas. Nestas, moravam 3344 pessoas, sendo $1635(48,9 \%)$ do sexo masculino e 1709 $(51,1 \%)$ do sexo feminino.

$\mathrm{O}$ inquérito familiar foi respondido pelos pais em $733(83,7 \%)$, pelos filhos em 92 $(10,5 \%)$ e por outros membros da família em $50(5,8 \%)$ casas.

As casas de Lagamar possuem, em média, cinco cômodos. O número de moradores por casa variou entre um e 15 sendo, em média, quatro. As condições de moradia foram consideradas razoáveis em $842(96,2 \%)$ das casas. Em 860 (98,3\%) moradias havia água encanada fornecida pela rede de serviços públicos do munícipio.

A deposição das excretas era feita no vaso sanitário em $726(83 \%)$ das casas, em fossa em $83(9,4 \%)$ e em ambos, de forma indistinta, em $48(5,5 \%)$. O lixo doméstico, em $353(40,3 \%)$ casas, era recolhido pelo serviço da Prefeitura Municipal de Lagamar; em 174 (19\%) era depositado em terrenos baldios; em 278 $(35,6 \%)$ era queimado e jogado no quintal da casa ou, então, em terreno baldio. Em 595 $(68 \%)$ casas visitadas, não havia animais domésticos. Em 177 (20,2\%) havia cães; em 60 $(6,9 \%)$ suínos; em $37(4,2 \%)$ cães e suínos e, em $3(0,3 \%)$ suínos e bovinos.

Com relação aos antecedentes sobre a criação de suínos, em $576(65,6 \%)$ casas, os informantes responderam negativamente, embora a maioria já os tivesse criado, quando morava em área rural; $193(22 \%)$ ja os tinham criado dentro da cidade e $100 \quad(11,4 \%)$ informaram que estavam criando, no momento, e já haviam criado no passado. Aqueles que criam suínos informaram que o número de animais existentes, no momento, variava entre um e 40 nos diferentes chiqueiros, tendo sido cadastrados um total de 406 animais.

Em 49 (49\%) das 100 casas com chiqueiro, o tempo que estavam criando os animais oscilava entre um e cinco anos e, em 21 (21\%), esse tempo chegava até 45 anos.

Em $593(67,8 \%)$ os informantes negaram identificar qualquer característica para reconhecer a doença nos suínos e, em 282 $(32,2 \%)$ a identificaram por uma ou várias de suas manifestações (Tabela 1). Dessas, a mais freqüente era o engrossamento muscular, definido como hipertrofia muscular da paleta do suíno pela infestação com cisticercos, mencionado por 220 (70\%) informantes, seguido pelo olho amarelado ou avermelhado, apontado por $94(29,7 \%)$ e atraso no desenvolvimento por 34 (12\%). Manifestações pouco usuais dos animais infectados, tais como tosse, tristeza etc, foram referidas por 24 $(8,5 \%)$ informantes.

Os chiqueiros localizavam-se geralmente no peridomicílio, sendo as condições de construção e manutenção extremamente precárias, o que favorecia o contato dos suínos com os moradores. Sobre esse fato, vale à pena assinalar que, desde o início do inquérito, detectamos a existência de um laticínio dentro da cidade, que comercializava o leite da região e se desfazia do soro, através de um "soroduto" que desaguava no riacho, que atravessava a cidade. Em grande parte, o soro era aproveitado pelos moradores que faziam fila, diariamente, para coletá-lo. Esse 
Silua-Vergara ML, Prata A, Vieira CO, Castro JH, Micheletti LG, Otaño AS, Franquini Jünior J. Aspectos epidemiológicos da teniase-cisticercose na área endêmica de Lagamar, MG. Revista da Sociedade Brasileira de Medicina Tropical 28:345-349, out-dez, 1995.

Tabela 1 - Forma popular de reconhecer a cisticercose nos suinos em Laganar/MG.

\begin{tabular}{lrr}
\hline \multicolumn{1}{c}{ Características dos animais } & $\mathrm{N}^{2}$ & $\%$ \\
\hline Engrossamento muscular & 145 & 51,4 \\
Atraso no desenvolvimento & 13 & 4,6 \\
Olho vermelho ou amarelo & 23 & 8,1 \\
Engrossamento muscular + atraso no desenvolvimento & 16 & 5,6 \\
Engrossamento muscular + olho vermelho ou amarelo & 54 & 19,1 \\
Atraso no desenvolvimento + olho vermelho ou amarelo & 2 & 0,7 \\
Engrossamento muscular + atraso no desenvimento + olho vermelho ou amarelo & 5 & 1,7 \\
Outras formas & 24 & 8,5 \\
Total & 282 & 100,0 \\
\hline
\end{tabular}

soro era destinado à criação dos suínos, os quais se mantinham bastante gordos, tornando, assim, mais rentável a criação para seus donos. Além disso, o soro que caía no riacho contaminava boa parte do seu trajeto e era, ali, justamente onde se concentrava a construção de muitos desses chiqueiros.

Antecedente de eliminação de proglotes, tênias ou diagnóstico de teníase, foi negado por $517(59,1 \%)$ informantes, enquanto 300 $(34,2 \%)$ referiram expulsão de vermes adultos ou proglotes ou diagnóstico de teníase em algum membro da família nuclear. Quanto ao número de membros afetados por teníase, no passado, havia $230(76,6 \%)$ casas com um membro da família, $56(18,6 \%)$ com dois, 9 $(3 \%)$ com três e $5(1,6 \%)$ com mais de três. A época mencionada da última expulsão de proglotes foi muito variável (Tabela 2 ).

Tabela 2 - Época da última expulsão de proglotes de Taenia spp pelos habitantes, em Lagamar/MG.

\begin{tabular}{ccc}
\hline Epoca de expulsão (anos) & $N^{2}$ & $\%$ \\
\hline$<1$ & 35 & 11,6 \\
$1-5$ & 61 & 20,3 \\
$6-10$ & 43 & 14,3 \\
$11-15$ & 38 & 12,6 \\
$>16$ & 123 & 41,0 \\
\hline Total & 300 & 100,0 \\
\hline
\end{tabular}

A informação sobre tratamento anterior para teníase foi referida por $141(16,1 \%)$ dos informantes, porém observamos confusão com respeito aos diferentes vermífugos recebidos.

Quanto à história pregressa de convulsôes, obtivemos a seguinte informação: em 749 $(85,6 \%)$ casas os informantes negaram ter algum membro afetado, no passado ou atualmente, e em $125(14,2 \%)$ casas o antecedente de convulsão foi positivo, havendo somente um membro afetado em
107. As convulsões tinham se iniciado tardiamente em $39(37,8 \%)$ pessoas dentro das famílias inqueridas. Antecedentes de doenças mentais na família foram referidos por 52 $(6 \%)$ informantes, não sendo possível a caracterização específica de tais doenças. $O$ exame parasitológico de fezes, realizado em $1850(55,3 \%)$ pessoas, revelou $24(1,3 \%)$ portadores de Taenia spp.

\section{DISCUSSÃO}

O simples fato de a terça parte da população de Lagamar conhecer as manifestações de teníase nos suínos serve de indicador para a existência da doença na região. O abate dos animais é realizado de forma clandestina, como costuma acontecer na maioria das áreas urbanas e rurais mais afastadas dos grandes centros 5789 . O fato de negarem a presença atual da cisticercose nos animais, mesmo quando se insistiu sobre o assunto, talvez resulte no receio da população quanto à interferência sanitária na criação dos animais, com seu sacrifício e proibição na comercialização da carne.

Além disso, a população possui conhecimento razoável sobre as conseqüencias do alojamento da larva da tênia, em regiōes como o sistema nervoso central. A criação de suínos dentro da cidade, de forma muito primitiva, favorece, sem dúvida, o contato estreito destes animais com as pessoas.

Das autoridades de saúde do município obtivemos a informação de que o abastecimento de água, a rede de esgostos e a coleta de lixo foram incrementados nos últimos anos. Recentemente, tem-se coibido, embora timidamente, a criação de suínos dentro da cidade. Pelas informações, acreditamos que a situação epidemiológica, alguns anos atrás, era ainda mais precária, 
Silva-Vergara ML, Prata A, Vieira CO, Castro JH, Micheletti LG, Otaño AS, Franquini Jünior J. Aspectos epidemiológicos da teníase-cisticercose na área endêmica de Lagamar, MG. Revista da Sociedade Brasileira de Medicina Tropical 28:345-349, out-dez, 1995.

quando suínos e pessoas confundiam-se nas ruas. Assim sendo, é possível presumir que essa população já tenha sofrido o peso maior dos fatores de risco associados à teníasecisticercose

O fato de, pelo menos, um membro da família, em 300 casas, ter expelido proglotes ou tênia adulta supera o que se observou em outros estudos similares ${ }^{79121617}$. Esse número nos pareceu tão elevado, que ficamos apreensivos sobre a possibilidade da superestimação, pela população, para obter preferência no atendimento médico que fazíamos concomitantemente com a realização do inquérito.

Quanto à cisticercose, para avaliar sua prevalência no inquérito epidemiológico, procuramos nos basear nas convulsões pois, de acordo com a literatura 101516202324 , em áreas endêmicas de cisticercose, esta pode explicar até $50 \%$ das etiologias das crises convulsivas. A informação de que alguma pessoa em 125 $(14,5 \%)$ domicilios tinha história de convulsão representa valor muito elevado. Às vezes, é muito difícil avaliar e caracterizar os diversos sinais e sintomas de convulsão, uma vez que existem inúmeras patologias e síndromes clínicas que podem causá-la ou simulá-la. Contudo, os dados obtidos foram reforçados pela avaliação clínico-neurológica desses pacientes ${ }^{22}$, pela experiência clínica dos médicos da cidade e pelo consumo exagerado de medicamentos anticonvulsivantes pela população.

A prevalência de $1,3 \%$ de ovos de Taenia $s p p$ nas fezes é baixa, mas está de acordo com outros inquéritos realizados em diversos locais ${ }^{8} 91617$. Contudo, mesmo essa porcentagem baixa, desde que haja, concomitantemente, a criação primitiva dos suínos, é suficiente para perpetuar a teníasecisticercose numa área, como tem sido ratificado em recentes trabalhos ${ }^{27891213182021}$.

\section{SUMMARY}

An epidemiological inquiry of bumancysticercosis due to Taenia solium was carried out in Lagamar, Minas Gerais State, Brazil, in 1992. A survey of 1109 bouses with 3344. inbabitants was made. The inquiry included 875 (86\%) families and the questionaire was answered by an informer, who was the fatber in $80 \%$ of the cases. One bundred pigsties, sbeltering 406 swines in extremely precarious conditions, were found in 100 (11.4\%) bouses. A bistory on taeniasis in some member of the family was verified in 300 (34.2\%) bouses. $A$ bistory of seizures was referred to by 125 (14.2\%) of families. The outset of convulsion in adult age was characterized in 39 (37.8\%) families. A bistory of mental disorder was reported in 53 (6.0\%) of bouses. Stool examinations were positive for Taenia spp in 24 (1.3\%) of samples examined.

Key-words: Taeniasis. Cysticercosis. Seizures. Epidemiological inquiry.

\section{AGRADECIMENTOS}

Ao Sr. Jovelino Cândido, Secretário de Saúde Municipal e à Prefeitura Municipal de Lagamar, por permitir-nos a realização deste trabalho. A população de Lagamar por aceitar participar deste inquérito.

\section{REFERÊNCIAS BIBLIOGRÁFICAS}

1. Arruda WO. Etiology of epilepsy: a prospective study of 210 cases. Arquivos de Neuro-Psiquiatria 49:251-254,1991.

2. Arruda WO, Camargo NJ, Coelho RJ. Neurocysticercosis: An epidemiological survey in two small rural communities. Arquivos de NeuroPsiquiatria 48:419-424,1990.

3. Beaver PC, Jung R, Cupp E. Cestodos ó gusanos en forma de cinta del hombre. In: Beaver PC, Jung R, Cupp E (eds) Parasitologia Clínica. $2^{\mathrm{a}}$ edicion española, Salvat editores p.527-533,1990.

4. Cook G. Neurocysticercosis: parasitological, clinical presentation, diagnosis, and recent advances in management. Quarterly Journal of Medicine 68:575-583,1988.

5. Cruz M, Davis A, Dixon H, Pawlowski ZK, Proano J. Operational studies on the control of Taenia solium taeniasis/cysticercosis in Ecuador. Bulletin of the World Health Organization 67:401407,1989 .

6. Davis A. A epidemiologia da teníase e da cisticercose. Jornal Brasileiro de Medicina 45:914,1983 .

7. Diaz F, Garcia HH, Gilman RH, Gonzalez AE, Castro M,Tsang WCW, Pilcher JB, Vasques LE, Lascano M, Carcamo C, Miranda E. and the cysticercosis working group in Peru. Epidemiology of taenisis and cysticercosis in a peruvian village. American Journal of Epidemiology 35:875-882,1992.

8. Diaz-Camacho $\mathrm{S}$, Candil-Ruiz A, Suate PV, Zazueta RM, Felix MM, Lozano R, Wilms K. Epidemiological study and control of Taenia solium infections with Praziquantel in a rural 
Silva-Vergara $M L$, Prata A, Vieira CO, Castro JH, Micheletti LG, Otaño AS, Franquini Jünior J. Aspectos epidemiológicos da teniase-cisticercose na ârea endêmica de Lagamar, MG. Revista da Sociedade Brasileira de Medicina Tropical 28:345-349, out-dez, 1995.

village of Mexico. The American Journal of Tropical Medicine and Hygiene 45:522-531,1991.

9. Diaz-Camacho S, Candil-Ruiz A, Uribe MB, Willms $\mathrm{K}$. Sorology as indicator of Taenia solium tapeworm infections in a rural community in Mexico. Transactions of the Róyal Society of Tropical Medicine and Hygiene 84:563-566,1990.

10. Dumas PM, Grunitzky K, Belo M, Dabis F, Deniau M, Bouteille B, Kassankogno Y, Catanzano G, Pestre AM. Cysticercose et neurocysticercose enquête épidemiologique dans le nord du Togo. Bulletin de la Société de Pathologie Exotique 83:263-274,1990.

11.Flisser A. Neurocysticerosis in México. Parasitology Today 4:131-136,1988.

12. Franco SS, Hincapié MC, Mejia O, Botero D. Estudio epidemiologico de epilepsia y neurocisticercosis. Revista Uis Medicina Bucaramanga 14:129-141,1986.

13. Gonzales Luarca E. Situação atual do complexo teníase humana-cisticercose nas Américas. Comunicações Científicas da Faculdade de Medicina Veterinária de São Paulo 8:222-226, 1984.

14. Mahajan RC. Geographical distribution of human cysticercosis In: Flisser A, Willms $\mathrm{K}$, Laclete JP, Larralde C, Ridaura C, Beltrán F (eds) Cysticercosis present state of knowledge and perspectives. N.Y Academic Press, New York p. 39-46, 1982.

15. Medina MT, Rosas E, Rubio Donnadieu F, Sotelo J. Neurocysticercosis as the main cause of lateonset epilepsy in Mexico. Archives of Internal Medicine 150:325-327,1990.

16. Sarti-Gutierres E, Shantz PM, Lara-Aguilera R, Gomez-Dandoy $\mathbf{H}$, Flisser A. Taenia solium taeniasis and cysticercosis in Mexican village. Annals of Tropical Medicine and Parasitology 39:194-198,1988.

17. Sarti-Gutierrez E, Shantz PM, Plancarte A, Wilson M, Gutierrez I, Lopez A, Robers J, Flisser A.
Prevalence and risk factors for Taenia solium taeniasis and cysticercosis in humans and pigs in a village in Morelos, Mexico. The American Journal of Tropical Medicine and Hygiene 46:677685, 1992.

18. Schenone H, Ramirez R, Rojas A. Aspectos epidemiologicos de la neurocisticercosis en America Latina. Boletin Chileno de Epidemiologia 28:61-72, 1973.

19. Schenone H, Villaroel F, Rojas A, Ramirez R. Epidemiology of human cysticercosis in Latin American. In: Flisser A, Willms $\mathbf{K}$, Laclete JP, Larralde C, Ridaura C, Beltrán F (eds) Cysticercosis present state of knowledge and perspectives. N.Y Academic Press, New York 2538, 1982.

20. Shorvon SD. Epidemiology, classification, natural history and genetics of epilepsy. The Lancet 836:93-96, 1990

21. Silva-Vergara ML. Aspectos epidemiológicos da teníase e contribuição ao estudo clínico da cisticercose na área endêmica de Lagamar/MG. Tese de Mestrado, Universidade de Brasília, Brasília, 1993.

22. Silva-Vergara ML, Vieira CI, Castro J, Micheletti L, Otaño A, Franquini Jr J, Cabral M, Leboreiro A, Marques J, Souza W, Costa-Cruz J, Prata A.Achados neurológicos e laboratoriais em população de área endêmica pata teníase-cisticercose, Lagamar; MG, Brasil (1992-1993). Revista do Instituto de Medicina Tropical de São Paulo 36:335-342, 1994.

23. Takayanagui OM, Jardim E. Aspectos clínicos da neurocisticercose: análise de 500 casos. Arquivos de Neuro-Psiquiatria 41:50-63, 1983.

24. Vianna LG, Macêdo V, Costa J, Mello P, Souza D. Estudo soroepidemiológico da cisticercose humana em Brasilia, Distrito Federal. Revista da Sociedade Brasileira de Medicina Tropical 19: 149156, 1986. 